\title{
Cathode priming of a relativistic magnetron
}

\author{
M. C. Jones, V. B. Neculaes, Y. Y. Lau, ${ }^{\text {a) }}$ R. M. Gilgenbach, and W. M. White \\ Intense Energy Beam Interaction Laboratory, Department of Nuclear Engineering \\ and Radiological Sciences, University of Michigan, Ann Arbor, Michigan 48109-2104
}

(Received 27 October 2004; accepted 8 December 2004)

A cathode priming technique of a relativistic magnetron is analyzed via a three-dimensional particle-in-cell simulation. By imposing a threefold azimuthal variation on the emitting cathode of a six-cavity relativistic magnetron, the electrons are prebunched at birth. This leads to fast startup of the pi mode with three electron spokes. Suppression of unwanted modes during startup is observed in the simulation. (C) 2004 American Institute of Physics. [DOI: 10.1063/1.1841454]

Priming of a radiation source always involves some external means by which the desired operating mode is preferentially excited. At least three objectives could be met with priming: (a) faster oscillation startup, (b) elimination of mode competition, and (c) frequency locking. The most obvious priming method is to inject a low level external signal at the same frequency of the desired operating mode. To a large degree, this seeding bypasses the buildup of oscillation from noise. In fact, radio frequency priming is an established technique for magnetrons and has recently been applied to a high power magnetron. ${ }^{1}$ The relativistic magnetron remains an important high power (gigawatt) microwave source. ${ }^{2-4}$

In this letter, we explore a class of priming techniques that is particularly suitable for the relativistic magnetron. Instead of injecting an external rf signal, we prepare the cathode $^{5}$ in such a way that its emission geometry favors excitation of the pi mode, the usual operating mode of the relativistic magnetron. We call this technique "cathode priming". It is much simpler and less expensive than rf priming.

For cathode priming of a six-cavity magnetron operating in the pi mode (three electron spokes), the cathode has three, azimuthally periodic, emitting regions around the cathode. Thus, a threefold symmetry in the electron bunches, which is a prerequisite of the pi mode, is immediately formed from the beginning. Such a cathode has been fabricated by ablating a pattern on the cathode by a $\mathrm{KrF}$ laser. ${ }^{5}$ This projectionablation-lithography cathode was installed in a relativistic magnetron, whose initial performance is reported elsewhere. In this letter, we present three-dimensional (3D) simulation results on this method of cathode priming which shows faststartup, suppression of extraneous modes during startup, and mode locking.

Figure 1(a) shows the Titan built, University of Michigan relativistic magnetron, which oscillates in the $\pi$ mode at a frequency of $1 \mathrm{GHz}$. Experimental configurations and results can be found elsewhere. ${ }^{4}$ The simulation geometry of the relativistic magnetron is shown in Figs. 1(b) and 1(c). Comparing Figs. 1(a) and 1(b) two major differences can be seen between the actual and the simulation magnetrons. First the cathode is larger, the experimental cathode radius is $0.635 \mathrm{~cm}$ and the simulation cathode radius that was used was $1.905 \mathrm{~cm}$, three times the actual cathode radius. The second difference is the anode vanes; the experimental anode vane tips are rounded, whereas in the simulation geometry they are not. The reason for these differences is to minimize

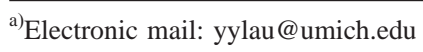

the computer runtimes. Accordingly, the Buneman-Hartree resonance condition has changed. The $\pi$-mode resonance for a voltage of $-300 \mathrm{kV}$ changed from $3 \mathrm{kG}$ with the experimental cathode radius $(0.635 \mathrm{~cm})$ to a value of $5 \mathrm{kG}$ for three times the cathode radius $(1.905 \mathrm{~cm})$. Figure 1(c) shows an axial cross section of the magnetron, including the cathode, cathode end caps, anode block, endplates on the anode block, as well as the outer shell of the magnetron. Cathode end caps were chosen to be 1.5 times the radius of the cathode; in the

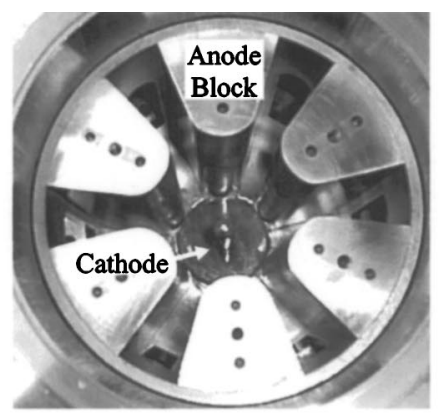

(a)

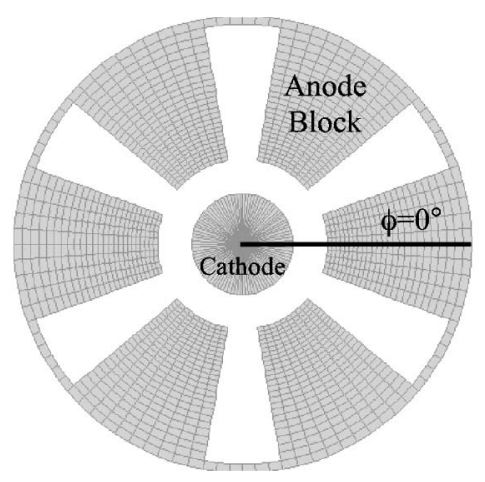

(b)

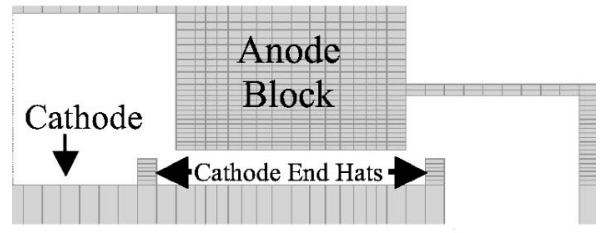

(c)

FIG. 1. (a) Geometry of A6 Titan relativistic magnetron with a cathode radius of $0.635 \mathrm{~cm}$. (b) Cross section of the simulation geometry, and (c) axial cross section of the simulation geometry; the axial length of the anode block is $20.5 \mathrm{~cm}$. 


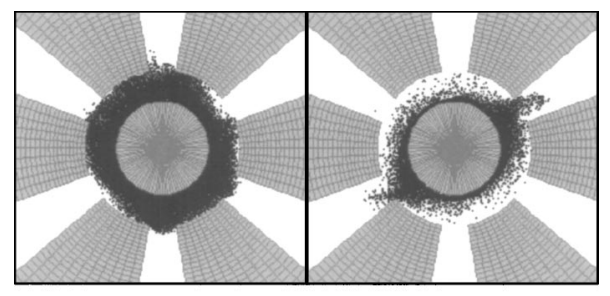

(a)

(b)

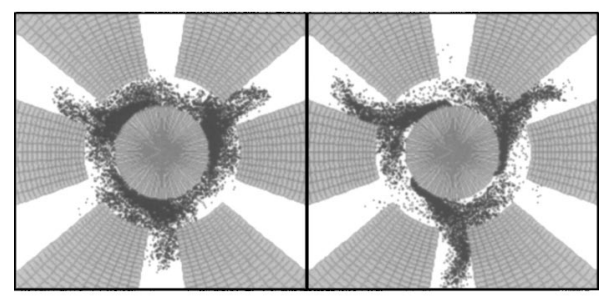

(c)

(d)

FIG. 2. Electron positions without cathode priming for (a) $t=7.363 \mathrm{~ns}$ and (b) $t=13.413 \mathrm{~ns}$. Electron positions with cathode priming (emission regions centered under the anode vanes). (c) $t=7.363 \mathrm{~ns}$ and (d) $t=13.413 \mathrm{~ns}$.

actual experiment the end caps are 2.54-cm-diam spherical aluminum spheres (two times the experimental cathode radius). The anode radius and the backwall radius are the same in both experiment and simulation.

Three different cases of cathodes were simulated using the 3D particle-in-cell, electromagnetic code, MAGIC: ${ }^{6}$ (1) one electron emission region with an axial length of $7 \mathrm{~cm}$, (2) three emission regions centered over the cavities of the magnetron with an axial length of $14 \mathrm{~cm}$, and (3) three emission regions centered over the anode vanes with an axial length of $14 \mathrm{~cm}$. For the cases with three electron emission regions, the emitting regions and nonemitting regions all had the same area, and the total emitting area was the same for all three cathodes used. The emission patches are binary, at full Child-Langmuir current or off. Explosive electron emission was limited to regions on the cathode between $-30^{\circ}$ to $30^{\circ}, 90^{\circ}$ to $150^{\circ}$, and $-150^{\circ}$ to $-90^{\circ}$ for the case with the emission centered under the vanes [Fig. 1(b), where the zero degree axis is marked]. Emission was limited between $0^{\circ}$ to $60^{\circ}, 120^{\circ}$ to $180^{\circ}$, and $-60^{\circ}$ to $-120^{\circ}$ for case with emission centered under the cavities of the magnetron. To characterize the startup and mode purity many diagnostics were taken: the rf electric field at the cavity entrance, electron phase space plots, emitted current, collected current, and average radius of electrons.

Presented in Figs. 2(a) and 2(c) are the electron positions at a time of $7.363 \mathrm{~ns}$ with (a) no priming and (c) with cathode priming with emission regions centered under the anode vanes. The benefits of the cathode priming are obvious, in that the primed magnetron is operating in the $\pi$ mode (three electron spokes for a six-cavity magnetron). At the time step of $13.413 \mathrm{~ns}$, the electrons in the magnetron with no cathode priming exhibit the characteristics of the $2 \pi / 3$ mode (two electron spokes in a six-cavity magnetron) shown in Fig. 2(b). In the case with cathode priming, shown in Fig. 2(d), the magnetron is still operating in the desired $\pi$ mode. Thus, the $2 \pi / 3$ mode is suppressed during startup by cathode priming.

Another essential tool to observe is the azimuthal rf electric field located at a center of a cavity. From this diagnostic

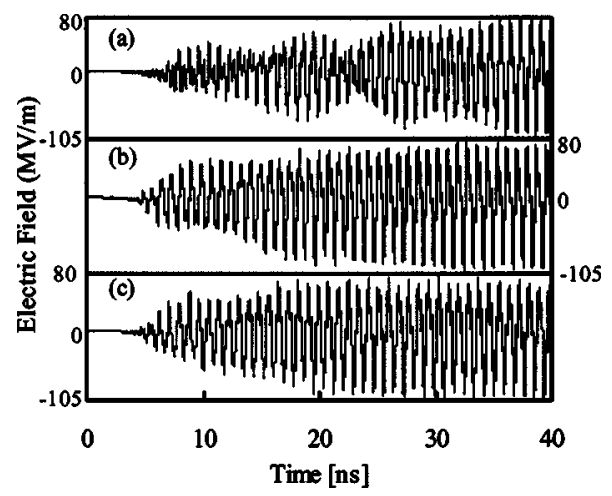

FIG. 3. rf electric field measured at the center of a cavity vs time. (a) No cathode priming, (b) cathode priming with emission regions located under the anode vanes, and (c) cathode priming with emission regions located under the cavities of the magnetron. Same vertical scales in (a)-(c).

alone, startup time and mode purity can be observed. Shown in Fig. 3 is the rf electric field versus time for the three different cases of cathodes. The maximum amplitude of the rf electric field is approximately the same for all three cases, implying that the output rf power is preserved with cathode priming. Trace (a) in Fig. 3 shows the rf field at the center of a cavity for the single emission cathode; an important note here is that the fields begin to be locked into the $\pi$ mode at a time of roughly $28 \mathrm{~ns}$ (see Fig. 4). It takes half that time for the rf fields to be locked into the $\pi$ mode for the cases with cathode priming; for the case with the emission regions located under the cavities it takes $13 \mathrm{~ns}$ for $\mathrm{rf}$ fields to be locked in the $\pi$ mode [trace (c) of Fig. 3], and $15 \mathrm{~ns}$ for trace (b) of Fig. 3. To find when the fields are locked in the desired $\pi$ mode, the instantaneous frequency was found from the azimuthal electric field. Figure 4 shows the instantaneous frequency for the rf fields shown in Fig. 3. The case with no cathode priming in Fig. 4 shows that beyond $30 \mathrm{~ns}$ the frequency does not vary more than $20 \mathrm{MHz}$ for the remainder of the simulation runtime. With cathode priming, and emission regions centered under the cavities, the frequency is locked into $\pi$ mode at roughly $10 \mathrm{~ns}$, after this time the frequency does not vary more than $20 \mathrm{MHz}$. Finally, with cathode priming and emission centered under the vanes, the instantaneous frequency of the azimuthal electric field is locked around $13 \mathrm{~ns}$. Also note that between 8 and $24 \mathrm{~ns}$, the rf fields with no cathode priming in Fig. 4 are operating at

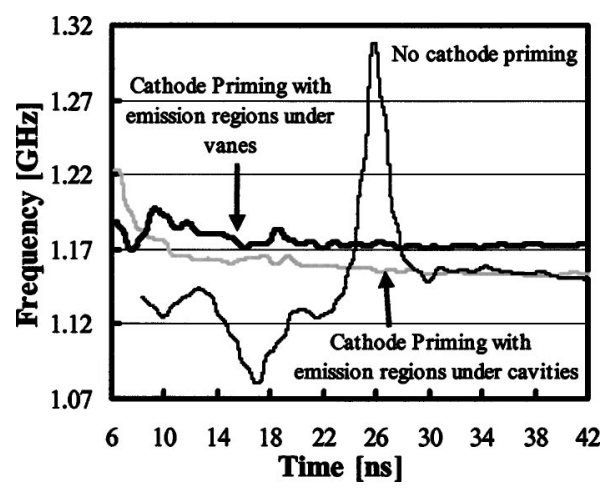

FIG. 4. Instantaneous frequency vs time for (a) no cathode priming, (b) cathode priming with emission regions located under the anode vanes, and (c) cathode priming with emission regions located under the cavities of the magnetron. 


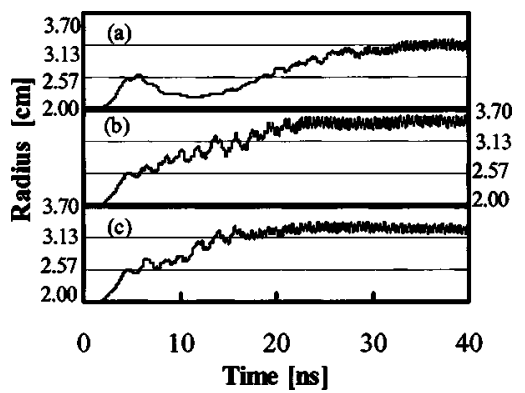

FIG. 5. Average radius of the electrons vs time for (a) no cathode priming, (b) cathode priming with emission regions located under the anode vanes, and (c) cathode priming with emission regions located under the cavities of the magnetron.

lower frequency, the $2 \pi / 3$ mode, which was seen in Fig. 2(b).

Another useful tool is to examine the average radius of the electrons in the magnetron as a function of time. Figure 5 shows that the expansion of the electron cloud is the same for all three cases between 2 and $5 \mathrm{~ns}$. After $5 \mathrm{~ns}$, for the case with no cathode priming, the radius of the electrons recedes and then reaches its steady-state value around $26 \mathrm{~ns}$, as shown in trace (a) of Fig. 5. For the case with cathode priming and emission centered over the vanes, trace (b) of Fig. 5, the radius of the electrons continue to expand after $5 \mathrm{~ns}$ and reaches its steady state value around $20 \mathrm{~ns}$. Finally, for the case of cathode priming and emission centered over the cavities, the electron radius reaches its steady-state value around 16 ns. From Fig. 5 it is shown that with cathode priming the radius of the electrons reach its steady-state value up to $50 \%$ faster.

Recently, we have invented the method of "magnetic priming", ${ }^{7-9}$ where an $N / 2$ azimuthal variation in the axial magnetic field is introduced in an $N$-cavity magnetron operating in the pi mode (N/2 electron spokes). There is no azimuthal variation in the cathode emission. This magnetic priming technique has been shown to lead to fast start-up, and most spectacularly, to significant noise reduction in microwave oven magnetrons. This was demonstrated in experiments $^{7,8}$ and confirmed to a certain degree by simulations. ${ }^{9,10}$ It is interesting to note that the reduction in the startup time is about $50 \%$, in both simulations of magnetic priming ${ }^{9,10}$ and of cathode priming, as shown in this paper. In principle, magnetic priming may also be applied to the relativistic magnetron. However, the large size of the relativistic magnetron makes magnetic priming more difficult experimentally compared with cathode priming. Our cathode priming technique 5 also leads to less severe gap closure in a relativistic magnetron.

Finally, we remark that azimuthal variations in cathode emission is usually undesirable, ${ }^{11}$ as it leads to mode competition and/or degradation of efficiency in virtually all beam-driven vacuum electron devices, such as gyrotron, klystron, and free electron laser. Here we introduce systematic variations on cathode emission, by design, so as to prebunch the electrons into the desired mode. The success of this cathode priming technique is due to two unique properties of magnetron: (a) the cathode and interaction region are one and the same, and (b) the quality of the electron "beam" during its formation is far less critical than any beam-driven device.

This research was supported by AFOSR and by the AFOSR/MURI Program on Breakdown and Emission in High Power Microwave Sources. The authors appreciate AFOSR support of the MAGIC Users Group provided by Mission Research Corporation.

${ }^{1}$ J. Robinson, M. Doherty, B. Davenport, M. Lander, and T. Treado, Proceedings of 3rd IEEE International Vacuum Electronics Conference, Monterey, CA, 23-25, April 2002, IEEE, Piscatawy, NJ, p. 24.

${ }^{2} \mathrm{~J}$. Benford and J. Swegle, High Power Microwaves (Artech House, Norwood, MA, 1992).

${ }^{3}$ R. W. Lemke, T. C. Genoni, and T. A. Spencer, IEEE Trans. Plasma Sci. 28, 887 (2000).

${ }^{4}$ M. R. Lopez, R. M. Gilgenbach, D. W. Jordan, S. A. Anderson, M. D. Johnston, M. W. Keyser, H. Miyake, C. W. Peters, M. C. Jones, V. B. Neculaes, Y. Y. Lau, T. A. Spencer, J. W. Luginsland, M. D. Haworth, R. W. Lemke, and D. Price, IEEE Trans. Plasma Sci. 30, 947 (2002); 32, 1171 (2004).

${ }^{5}$ M. C. Jones, V. B. Neculaes, R. M. Gilgenbach, W. M. White, M. R. Lopez, and Y. Y. Lau, Rev. Sci. Instrum. 75, 2976 (2004).

${ }^{6}$ B. Goplan, L. Ludeking, D. Smithe, and G. Warren, Comput. Phys. Commun. 87, 54 (1995).

${ }^{7}$ V. B. Neculaes, R. M. Gilgenbach, and Y. Y. Lau, Appl. Phys. Lett. 83, 1938 (2003).

${ }^{8}$ V. B. Neculaes, R. M. Gilgenbach, Y. Y. Lau, M. C. Jones, and W. M. White, IEEE Trans. Plasma Sci. 32, 1152 (2004).

${ }^{9}$ M. C. Jones, V. B. Neculaes, W. M. White, Y. Y. Lau, and R. M. Gilgenbach, Appl. Phys. Lett. 84, 1016 (2004).

${ }^{10}$ J. W. Luginsland, Y. Y. Lau, V. B. Neculaes, R. M. Gilgenbach, M. C. Jones, M. H. Frese, and J. J. Watrous, Appl. Phys. Lett. 84, 5425 (2004).

${ }^{11}$ J. P. Anderson, S. E. Korbly, R. J. Temkin, M. A. Shapiro, K. L. Felch, and S. Cauffman, IEEE Trans. Plasma Sci. 30, 2117 (2002). 